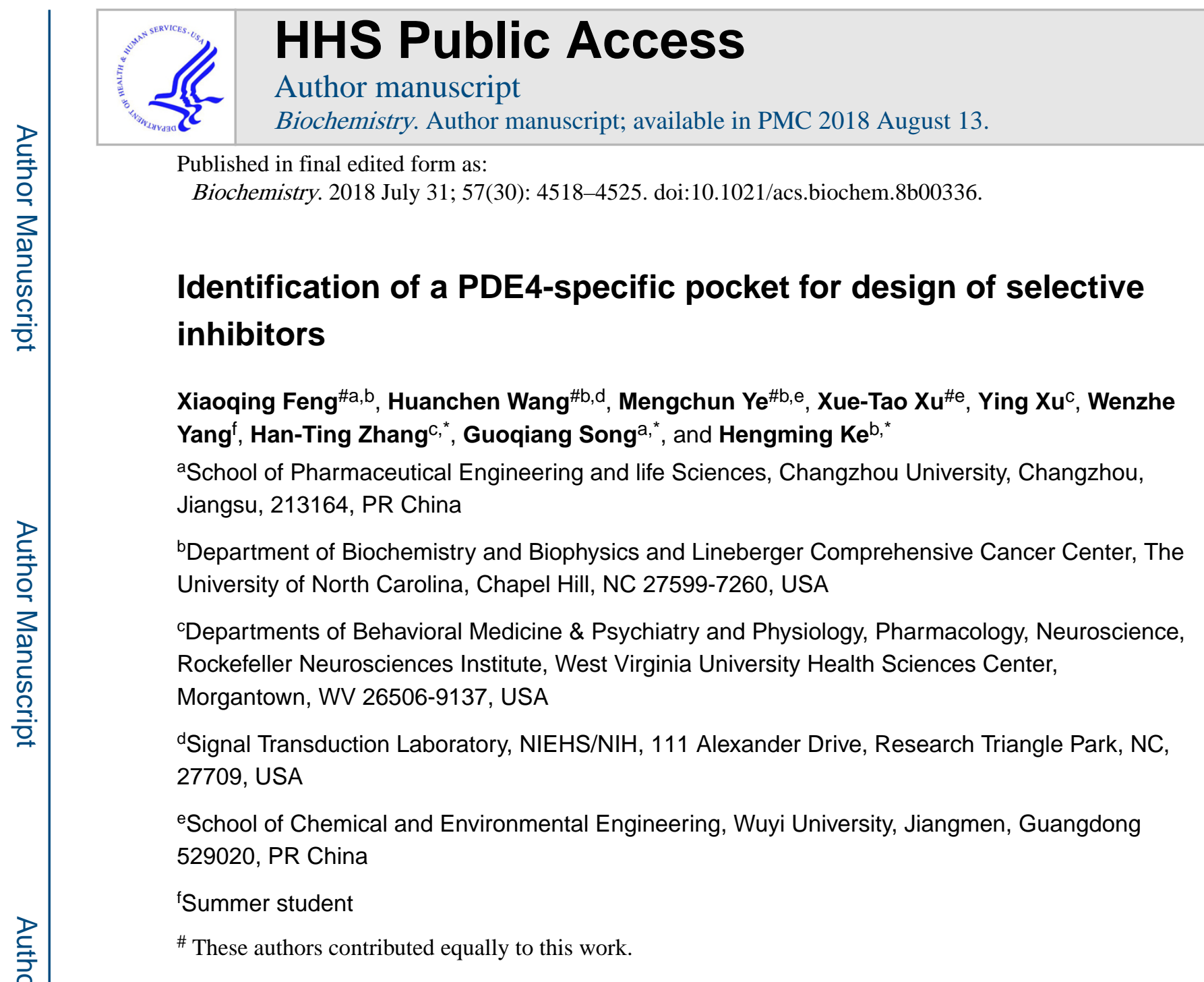

\title{
Abstract
}

Inhibitors of phosphodiesterases (PDEs) have been widely studied as therapeutics for treatment of human diseases, but improvement on inhibitor selectivity is still desirable for enhancement of inhibitor potency. Here, we report identification of a water-containing subpocket as a PDE4specific pocket for inhibitor binding. We designed against the pocket and synthesized two enantiomers of PDE4 inhibitor Zl-n-91. The (S)-Zl-n-91 enantiomer showed the $\mathrm{IC}_{50}$ values of 12 and $20 \mathrm{nM}$ respectively for the catalytic domains of PDE4D2 and PDE4B2B, thousand folds of selectivity over other PDE families, and potent neuroprotection activities. Crystal structures of the PDE4D2 catalytic domain in complex with each Z1-n-91 enantiomer revealed that (S)-, but not $(R)$-Zl-n-91, formed a hydrogen bond with the bound water in the pocket, thus explaining its higher affinity. The structural superposition between the PDE families revealed that this watercontaining subpocket is unique to PDE4 and thus valuable for design of PDE4 selective inhibitors.

\footnotetext{
*Corresponding Authors Tel: 1-919-966-2244, hke@med.unc.edu (H.K.); Tel: 86-158-8633-0600, drugs@ vip.sina.com (G.S.); Tel: 1-304-293-1488, hzhang@hsc.wvu.edu (H.Z.).

Author Contributions

H. K, H. T. Z., G. S, H. W., and M. Y. were anticipated in research design. X. F., M. Y., H. W, X. X., and Y. X. performed enzyme expression, crystallization, inhibitor design and synthesis, and structure determination. W. Y. was involved in docking. H. K, H. W., H. T. Z, and G. S. contributed to the manuscript writing.

PDB ID Codes

The atomic coordinates and structure factors have been deposited into the RCSB Protein Data Bank with accession codes of 5WH5 and 5WH6.
} 


\section{Graphical TOC}

A bound water defines the PDE4 specific pocket

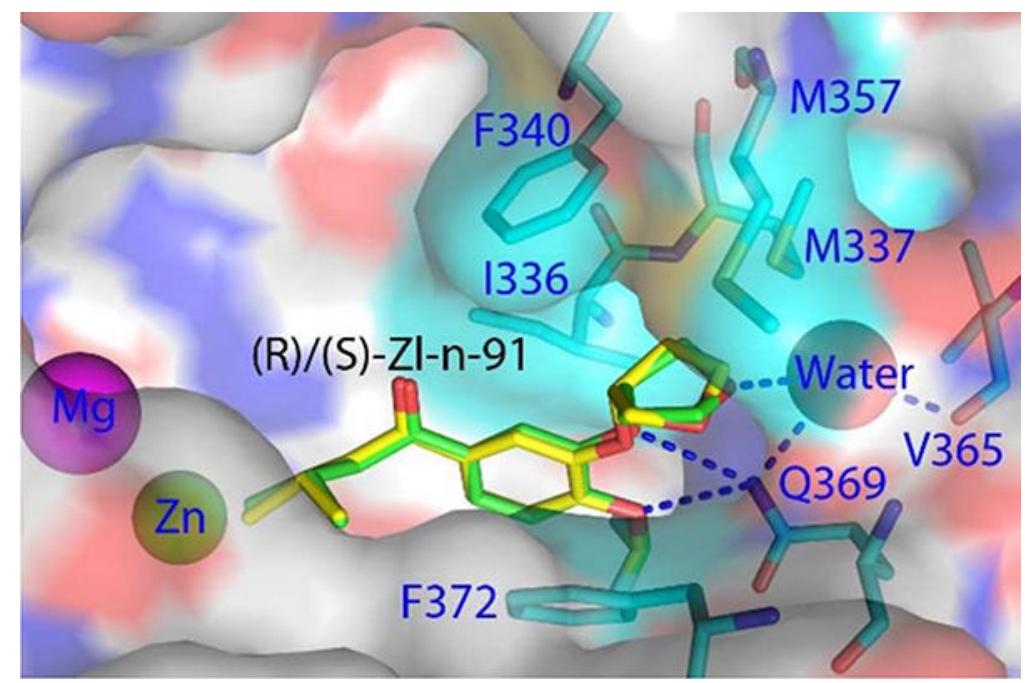

\section{Keywords}

Phosphodiesterase 4 selective inhibitor; a water pocket for inhibitor design; crystal structure of PDE4-inhibitor complex

Cyclic nucleotide phosphodiesterases (PDEs) are unique enzymes decomposing cellular cyclic adenosine and guanosine 3', 5'-monophosphate (cAMP and cGMP). ${ }^{1-3}$ The signaling systems of second messengers cAMP and cGMP represent the earliest signaling pathways and have received great attention in recent years for their critical roles in regulation of a vast number of physiological processes such as cardiovascular diseases. ${ }^{4}$ Human genome contains 21 PDE genes that are categorized into 11 families and expresses over 100 isoforms of PDE proteins through alternative mRNA splicing. ${ }^{1-3}$ PDE enzymes are divided into a variable regulatory domain at the $\mathrm{N}$-terminus and a conserved catalytic domain at the $\mathrm{C}$ terminus. The conserved catalytic domains of PDEs specifically recognize substrates and selectively bind inhibitors. PDE4, 7, and 8 preferably hydrolyze cAMP while PDE5, 6 and 9 are cGMP specific. The remaining PDE families $(1,2,3,10,11)$ show dual activity in hydrolysis of both cAMP and cGMP. ${ }^{1-3}$

Selective inhibitors of PDE4 have been extensively studied as anti-inflammatory drugs for treatment of asthma and chronic obstructive pulmonary disease (COPD). ${ }^{5}$ In addition, PDE4 inhibitors have been shown to be potential for treatment of many other diseases, including memory enhancement, ${ }^{6}$ inflammatory bowel disease, ${ }^{7}$ autoimmune diseases, ${ }^{8}$ respiratory and lung diseases, ${ }^{9}$ psoriasis, ${ }^{10}$ atopic dermatitis, ${ }^{11}$ Huntington's disease, ${ }^{12}$ neurological and psychiatric disorders, ${ }^{13}$ alcoholism, ${ }^{14}$ and Alzheimer's disease. ${ }^{15-17}$ The best known PDE4 inhibitor is roflumilast (Daxas, Daliresp, Fig. 1), which was approved as an orally administered drug for the treatment of severe COPD in Europe in June 2010 and in US in March 2011. ${ }^{18}$ Another PDE4 inhibitor, apremilast (Otezla) was approved by the United 
States Food and Drug Administration in 2014 for the treatment of active psoriatic arthritis and moderate to severe plaque psoriasisis. ${ }^{19}$ Recently, crisaborole (Eucrisa), a boroncontaining drug was approved by US FDA for the topical treatment of psoriasis and atopic dermatitis in December, 2016. ${ }^{20}$

Despite the fact that three PDE4 drugs are in market, the wide effects of PDE4 inhibitors on the neuro-system and other physiological processes may imply a possibility of new medical applications of PDE4 inhibitors. In addition, two current drugs of roflumilast and apremilast are lack of ability to penetration brain and have dose-limited side effects such as emesis. Thus, improvement on selectivity of PDE4 inhibitors over other PDE families, especially discovery of PDE4B subfamily-selective inhibitors are desirable for reduction of side effects and development of new applications. ${ }^{21-23}$ Here, we conducted structural and enzymatic studies of the PDE4D2 catalytic domain in complex with each enantiomer of inhibitor 1-(4(difluoromethoxy)-3-((tetrahydrofuran-3-yl)oxy)phenyl)-3-methylbutan-1-one (Zl-n-91). The structures revealed a water-containing subpocket that appears to be unique to PDE4 families. This piece of information is valuable for design and development of PDE4 inhibitors.

\section{Experimental methods}

\section{Synthesis of (S)- and (R)-ZI-n-91}

The synthetic route for $(S)$ - and $(R)$-Z1-n-91 is shown in Fig. 2.

Compound 2: (4-Difluoromethoxy-3-hydroxybenzaldehyde). ${ }^{24}$-To a solution of 3,4-hydroxybenzaldehyde (1, Fig. 2, $1.38 \mathrm{~g}, 10 \mathrm{mmol})$ in DMF (20 mL), methyl chlorodifluoroacetate $(1.39 \mathrm{~mL}, 13 \mathrm{mmol})$ and cesium carbonate $(4.23 \mathrm{~g}, 13 \mathrm{mmol})$ were added. After stirred at $70^{\circ} \mathrm{C}$ for $3 \mathrm{~h}$ under air atmosphere, DMF was removed in vacuo. The residue was portioned between aqueous $3 \mathrm{~N} \mathrm{HCl}$ and ethyl acetate. Layers were separated and the aqueous layer was extracted with ethyl acetate $(3 \times 30 \mathrm{~mL})$. Combined organic layers were washed with brine, dried over $\mathrm{Na}_{2} \mathrm{SO}_{4}$, and concentrated in vacuo. The crude product was purified by flash chromatography (Hexane:EtOAc $=5: 1$ ) to afford compound 2 $(0.61 \mathrm{~g}, 33 \%)$ as a white solid. ${ }^{1} \mathrm{H}$ NMR $\left(400 \mathrm{MHz}, \mathrm{CDCl}_{3}\right) \delta 9.92(\mathrm{~s}, 1 \mathrm{H}), 7.55(\mathrm{~d}, J=4.0$ $\mathrm{Hz}, 1 \mathrm{H}), 7.45$ (dd, $J=4.0,8.4 \mathrm{~Hz}, 1 \mathrm{H}), 7.28(\mathrm{~d}, J=8.4 \mathrm{~Hz}, 1 \mathrm{H}), 6.67$ (t, $J=72.8 \mathrm{~Hz}, 1 \mathrm{H})$, 6.17 (br s, 1H).

\section{Compound 3: (S)- or (R)-(3-((Tetrahydrofuran-3-yl)oxy)-4-} (difluoromethoxy)benzaldehyde). ${ }^{25}$-To a $0{ }^{\circ} \mathrm{C}$ solution of $\mathrm{PPh}_{3}(723 \mathrm{mg}, 2.76 \mathrm{mmol})$ and $(R)$ - or (S)-3-hydroxytetrahydrofuran $(223 \mu \mathrm{L}, 2.76 \mathrm{mmol})$ in $\mathrm{CH}_{2} \mathrm{Cl}_{2}(10 \mathrm{~mL})$, was added dropwise with syringe to a solution of di-tert-butyl azodicarboxylate $(636 \mathrm{mg}, 2.76$ mmol) in $\mathrm{CH}_{2} \mathrm{Cl}_{2}(5 \mathrm{~mL})$. After 5 minutes, a solution of compound 2 in $\mathrm{CH}_{2} \mathrm{Cl}_{2}(5 \mathrm{~mL})$ was added dropwise with syringe. The reaction mixture was warmed to room temperature and stirred for $8 \mathrm{~h}$. After removal of the solvent, the residue was purified by flash chromatography (Hexane:EtOAc $=5: 1)$ to afford compound $(S)$ or $(R)-3(0.46 \mathrm{~g}, 96 \%)$ as a colorless oil. ${ }^{1} \mathrm{H}$ NMR $\left(400 \mathrm{MHz}, \mathrm{CDCl}_{3}\right) \delta 9.93(\mathrm{~s}, 1 \mathrm{H}), 7.48(\mathrm{dd}, J=1.6,8.4 \mathrm{~Hz}, 1 \mathrm{H})$, $7.44(\mathrm{~d}, J=1.6 \mathrm{~Hz}, 1 \mathrm{H}), 7.33$ (d, $J=8.4 \mathrm{~Hz}, 1 \mathrm{H}), 6.67$ (t, $J=74.4 \mathrm{~Hz}, 1 \mathrm{H}), 5.08-5.06$ (m, $1 \mathrm{H}), 4.06-3.91(\mathrm{~m}, 4 \mathrm{H}), 2.31-2.29(\mathrm{~m}, 1 \mathrm{H}), 2.20-2.18(\mathrm{~m}, 1 \mathrm{H})$. 


\section{Compound 4:(S)-or (R)-(I -(4-(Difluoromethoxy)-3-(tetrahydrofuran-3-} yloxy)phenyl)-3-methylbutan-1-ol). ${ }^{26}$-To a $-20^{\circ} \mathrm{C}$ solution of compound $(S)$ - or (R)-3 $(129 \mathrm{mg}, 0.5 \mathrm{mmol})$ in THF $(2 \mathrm{~mL})$, Grignard Reagent $(0.6 \mathrm{mmol})$ was added dropwise. The reaction mixture was stirred at $-20^{\circ} \mathrm{C}$ for $1.5 \mathrm{~h}$ and then $2 \mathrm{~mL}$ of $1 \mathrm{~N} \mathrm{HCl}$ was added to quench the reaction. The mixture was extracted with EtOAc and combined organic layers was washed with brine, dried over $\mathrm{Na}_{2} \mathrm{SO}_{4}$ and concentrated in vacuo to afford crude compound (S)- or (R)-4. ${ }^{1} \mathrm{H}$ NMR (400 MHz, $\left.\mathrm{CDCl}_{3}\right) \delta 7.13(\mathrm{~d}, J=8.4 \mathrm{~Hz}, 1 \mathrm{H}), 6.95(\mathrm{~s}$, $1 \mathrm{H}), 6.90(\mathrm{~d}, J=8.4 \mathrm{~Hz}, 1 \mathrm{H}), 6.51(\mathrm{t}, J=75.2 \mathrm{~Hz}, 1 \mathrm{H}), 5.00(\mathrm{~m}, 1 \mathrm{H}), 4.70-4.74(\mathrm{~m}, 1 \mathrm{H})$, 4.03-3.90 (m, 4H), 2.22-2.20 (m, 2H), 1.94 (br s, 1H), 1.72-1.70 (m, 2H), 1.48-1.46 (m, $1 \mathrm{H}), 0.96(\mathrm{~d}, J=6.0 \mathrm{~Hz}, 6 \mathrm{H})$.

Compound (S)- or (R)-ZI-n-9I: (S)- or (R)-(I-(4-(Dfluoromethoxy)-3(tetrahydrofuran-3-yloxy)phenyl)-3-methylbutan-I-one. ${ }^{27}$-To a solution of crude product $(S)$ - or $(R)-4$ in $\mathrm{CH}_{2} \mathrm{Cl}_{2}(3 \mathrm{~mL})$, solid $\mathrm{PCC}(216 \mathrm{mg}, 0.6 \mathrm{mmol})$ was added portionwise. After stirred at room temperature for $2 \mathrm{~h}$, the mixture was filtered (eluting with EtOAc) and the filtrate was concentrated in vacuo. The residue was purified by flash chromatography to afford enantiomeric (S)- or (R)-Zl-n-91. (S)- or (R)-Zl-n-91 at $1 \mathrm{~g} / \mathrm{L}$ concentration in DMSO has optical rotation values of $[a]_{D}=-0.15$ and 0.16 respectively for (S)- and (R)-Zl-n-91 at $25^{\circ} \mathrm{C} .{ }^{1} \mathrm{H}$ NMR $\left(400 \mathrm{MHz}, \mathrm{CDCl}_{3}\right) \delta 7.56-7.53(\mathrm{~m}, 2 \mathrm{H}), 7.23$ $(\mathrm{d}, J=8.8 \mathrm{~Hz}, 1 \mathrm{H}), 6.62(\mathrm{t}, J=74.4,1 \mathrm{H}), 5.10-5.03(\mathrm{~m}, 1 \mathrm{H}), 4.06-3.93(\mathrm{~m}, 4 \mathrm{H}), 2.80(\mathrm{~d}, J$ $=6.8 \mathrm{~Hz}, 2 \mathrm{H}), 2.32-2.18(\mathrm{~m}, 3 \mathrm{H}), 1.01(\mathrm{~d}, J=6.8 \mathrm{~Hz}, 6 \mathrm{H})$.

\section{Protein expression, crystallization and structure determination}

The protein expression and purification of the catalytic domains of PDE4 and other PDE families have been previously reported. ${ }^{28-32}$ About $10 \mathrm{mg} / \mathrm{ml}$ PDE4D2 (86-410) was stored in a buffer of $20 \mathrm{mM}$ Tris.base, $\mathrm{pH} 7.5,50 \mathrm{mM} \mathrm{NaCl}, 1 \mathrm{mM} \beta$-mercaptoethanol, $1 \mathrm{mM}$ EDTA and mixed with $2 \mathrm{mM}(. R)$ - or $(S)$-Zl-n-91. The PDE4D2 complexes were crystallized at $4^{\circ} \mathrm{C}$ by hanging drop against a well buffer of $0.1 \mathrm{M}$ HEPES $\mathrm{pH} 7.5,15 \%$ PEG3350, 30\% ethylene glycol, and 10\% isopropanol. The diffraction data were collected on beamline X29 at Brookhaven National Laboratory and processed by program HKL. ${ }^{33}$ The structures were solved by molecular replacement, using the wild type PDE4D2 as the initial model. ${ }^{34}$ The atomic model was rebuilt by program COOT $^{35}$ and refined by program REFMAC (Table 1). ${ }^{36}$

\section{Enzymatic assay.}

The enzymatic activities of Zl-n-91 were assayed using ${ }^{3} \mathrm{H}$-cAMP or ${ }^{3} \mathrm{H}$-cGMP as substrates, as previously reported. ${ }^{28}$ Briefly, the proteins were incubated with a reaction mixture of $20 \mathrm{mM}$ Tris. $\mathrm{HCl}, \mathrm{pH} 7.5,10 \mathrm{mM} \mathrm{MgCl} 2,0.5 \mathrm{mM}$ DTT, ${ }^{3} \mathrm{H}$-cAMP or ${ }^{3} \mathrm{H}$-cGMP (20-40k cpm/assay) at room temperature for $15 \mathrm{~min}$. The reaction was terminated by addition of $0.2 \mathrm{M} \mathrm{ZnSO}_{4}$. The reaction product ${ }^{3} \mathrm{H}$-AMP or ${ }^{3} \mathrm{H}-\mathrm{GMP}$ was precipitated out by addition of $0.2 \mathrm{~N} \mathrm{Ba}(\mathrm{OH})_{2}$ while unreacted ${ }^{3} \mathrm{H}$-cAMP or ${ }^{3} \mathrm{H}$-cGMP stayed in the supernatant. Radioactivity in the supernatant was measured by a liquid scintillation counter. The inhibition on PDEs was measured at nine concentrations of inhibitors, using suitable concentrations of the substrates and enzymes. Rolipram was used as a reference to be sure the accuracy of the measurement. 


\section{Measurement of pharmacokinetic property}

The pharmacokinetic properties of Zl-n-91 were analyzed by HD Biosciences Co., Ltd, Shanghai, China. The stock sample was prepared by suspension of $10 \mathrm{mg}$ Zl-n-91 in $10 \mathrm{ml}$ of saline solution containing $1 \%$ DMSO, $40 \%$ HP- $\beta$-CD (2-Hydroxypropyl-betacyclodextrin), and 6\% Solutol HS 15 (2-hydroxyethyl 12-hydroxyoctadecanoate). The mixture was vigorously vortexed and sonicated until the solid was completely dissolved. Compound Zl-n-91 was given to male ICR mice at dose of $5 \mathrm{mg} / \mathrm{kg}$ body weight.

\section{Behavioral tests for potential memory enhancement and sedative effect of ZI-n-91 in mice}

Male ICR mice (Harlan, Indianapolis, IN), weighing 22-24 g, were housed in a temperaturecontrolled room $\left(22-23^{\circ} \mathrm{C}\right)$ and maintained on a 12-h light-dark cycle (lights on at 6:00am). Water and food were freely available in the home cages. All experiments were carried out according to the NIH Guide for the Care and Use of Laboratory Animals (NIH Publications No. 80-23, revised 1996). The procedures were approved by the Animal Care and Use Committee of West Virginia University Health Sciences Center.

To determine the effect of Zl-n-91 on memory, we evaluated the performance of mice after treated with Z1-n-91, rolipram, or vehicle in the object recognition test. ${ }^{37}$ Mice were trained to discriminate a novel object from the familiar one in an arena with a 24-hour delay. The time spent in exploring the novel (Tn) and familiar (Tf) objects was recorded for 3 min and the discrimination ratio, (Tn-Tf)/(Tn+Tf), was calculated as an index of memory.

For determination of the potential sedative effect of Zl-n-91, we examined locomotor activity in the open-field test. ${ }^{38}$ Thirty minutes after treatment with different doses of Zl$\mathrm{n}$-91, rolipram, or vehicle, naive mice were individually placed in the center of the openfield box, with the floor divided into 4 identical squares, and allowed to explore for $5 \mathrm{~min}$. The number of line crossings (all four paws crossed the line) and rearing (i.e. the number of standing on the mouse rear) were recorded. All data were analyzed using one-way ANOVA, followed by post hoc Dunnett's tests. Statistical significance was accepted at $\mathrm{p}<0.05$.

\section{Results}

\section{A bound water in the inhibitor binding subpocket of PDE4}

The active site of PDEs is an open cavity and composes of a metal site, an inhibitor pocket and an open region (Fig. 3). The inhibitor binding pocket can be further divided into a hydrophobic clamp and two subpockets of Q1 and Q2. ${ }^{39}$ In PDE4D2, the catechol group of rolipram (Fig. 1), the first generation of PDE4 inhibitors, fits the hydrophobic clamp by interacting with the conserved residues of Ile336, Phe340, and Phe372, in addition to a hydrogen bond with invariant Gln369 (Fig. 3). These interactions, the hydrogen bond with Gln369 and the hydrophobic stack against Phe372, are conserved in all PDE families and contribute basic affinity to PDE inhibitors. ${ }^{40}$

Historically, the Q2 subpocket was treated as a hydrophobic site for binding of cilomilast and roflumilast (Fig. 1), in which the cyclic pentane of cilomilast and the propane ring of roflumilast occupied the pocket (Fig. 3). However, a careful examination on the PDE4 
structures (PDB codes of $1 \mathrm{XOM}$ and $1 \mathrm{XOQ}$ ) shows that a bound water molecule sits in the Q2 pocket and forms two hydrogen bonds with the side chain of Gln369 and the backbone carbonyl oxygen of Val365. It is interesting to note that the tetrahydrofuran ring of our previously published racemic Zl-n-91, ${ }^{41}$ which has $\mathrm{IC}_{50}$ of $26 \mathrm{nM}$ against PDE4D, might occupy this water-containing pocket for its similarity to cilomilast and roflumilast (Fig. 1). If so, either (S)- or (R)-Zl-n-91 would form a hydrogen bond with the water and may improve its affinity and selectivity. Thus, we synthesized (R)- and (S)-enantiomers of Zl-n-91 and determined the crystal structures of the PDE4D catalytic domain in complex with either enantiomer of Zl-n-91.

\section{Pharmacological properties of Zl-n-91}

Compound Z1-n-91 has small molecular weight of 314 Daltons and exists in a thick liquid form. It has $\log P$ of $2.18, \operatorname{LogS}$ of -3.08 , and tPSA of 44.8 , as calculated by ChemDraw. These chemical properties would imply its reasonable ability to penetrate cell membrane. The $(S)$ - and (R)-enantiomers have the $\mathrm{IC}_{50}$ values of 12 and $65 \mathrm{nM}$ for the catalytic domain of PDE4D2 (86-410), and 20 and $89 \mathrm{nM}$ for the catalytic domain of PDE4B2B (152-528), respectively. (S)-Zl-n-91 shows at least 1000-fold selectivity over other PDE families (Table 2). In the whole blood cell, Zl-n-91 inhibits the LPS-induced TNFa release with $\mathrm{IC}_{50}$ of 40 $\mathrm{nM}$, in comparison with $100 \mathrm{nM}$ for rolipram ${ }^{41}$ and $>10 \mu \mathrm{M}$ for the potent PDE4 inhibitor cilomilast, ${ }^{42}$ implying superior anti-inflammation activity of Zl-n-91.

Pharmacokinetic analysis using male C57/BL6 mice shows that Zl-n-91 has T1/2 of 1.35 hours, $T^{1 / 2}$ range of $4-8$ hours, and bioavailability of $33 \%$ in oral administration mode (Table 3). After 2 hours of oral administration, the plasma concentration of Zl-n-91 reached maximum of $96.5 \mathrm{ng} / \mathrm{mL}$ that is about 25 folds to its $\mathrm{IC}_{50}$ of $3.7 \mathrm{ng} / \mathrm{mL}$, suggesting a potential of Zl-n-91 as a drug lead.

\section{Binding of (R)- and (S)-enantiomers of ZI-n-91 to PDE4D}

The crystal structures show that (R)- and (S)-Zl-n-91 have essentially the same orientation and conformation, except for the hydrogen bond of (S)-Zl-n-91 with the bound water. The catechols of both Zl-n-91 enantiomers fit the hydrophobic slot, are clamped by Ile336 and Phe 340 on one side and Phe372 on another side, in addition to two hydrogen bonds with invariant Gln369 (Figs. 4A \& 4B). The $\mathrm{CF}_{2}$ groups of Zl-n-91s contact hydrophobic residues of Val168, Met273, and Leu319, while the tetrahydrofuran rings form van der Waals' interactions with Met337, Phe340, Met357, and Val365.

In the PDE4D2 structure, the oxygen of the tetrahydrofuran ring of (S)-Z1-n-91 forms a hydrogen bond with the water molecule that is fixed by the hydrogen bonds with the side chain of invariant Gln369 and the backbone oxygen of Val365 (Fig. 4B). It is interesting to note that a water molecule was also observed and occupied the same position in the pockets of PDE4A and 4B. ${ }^{43}$ The water forms hydrogen bonds with the corresponding residues Met549, Gln581 and Val577 of PDE4A10 (PDB code of 2QYK, Fig. 4C), and Met412, Gln443 and Val439 of PDE4B (PDB code of 2QYL, Fig. 4D). Unfortunately, disorder of invariant Gln491 in the PDE4C2 structure (PDB code of 2QYM) leaves it unknown if the corresponding water exists in PDE4C. Since this bound water is observed in the pocket of 
PDE4 but not other PDEs, we speculate that this pocket may serve as a PDE4 specific pocket for improvement of affinity and selectivity of PDE4 inhibitors, as discussed in the later section.

The sequence alignment of the PDE4 subfamilies showed that residues of the hydrophobic slot and the water pocket are absolutely conserved, including Ile336, Met337, Phe340, Met357, Val365, Ser368, Gln369, and Phe372 (PDE4D2 numbering). The structure superposition among PDE4D2, PDE4A10, and PDE4B2B (Fig. 4E) revealed minor conformation adjustment, except for Met357 and Val365, thus implying that inhibitors against this water-binding pocket may not be PDE4 subfamily-selective.

\section{Memory improvement of ZI-n-91}

To determine whether Zl-n-91 improves memory, we tested the object recognition of mice. The discrimination ratio (Tn-Tf)/(Tn+Tf), which is defined as the ratio of time for mice to recognize familiar (Tf) and novel ( $\mathrm{Tn}$ ) objects after trained with identical objects, reflects their memory in the object recognition test. ${ }^{37}$ Rolipram at $0.3 \mathrm{mg} / \mathrm{kg}$ dosage increased the discrimination ratio by about 4 times (Fig. 5A), while Zl-n-91 needed only $0.01 \mathrm{mg} / \mathrm{kg}$ $\left(\mathrm{F}_{3,33}=5.87, \mathrm{P}=0.003\right.$ by ANOVA $)$ to produce the same effect, suggesting that Zl-n-91 improves long-term memory at a $1 / 30$ dose of rolipram. However, at the higher dose of 0.1 $\mathrm{mg} / \mathrm{kg}$, Zl-n-91 increases the discrimination ratio by only 2-folds of the vehicle control. The explanation to this unusual dose-response is not clear, perhaps due to off-target effects. The effects of Zl-n-91 did not appear to be the consequence of potential sedation because locomotor activity was not altered, as discussed below.

For determination of the potential sedative side effect of Z1-n-91, we examined locomotor activity using the open-field test. As shown in Fig. 5B, Zl-n-91 at doses of 0.01-0.5 mg/kg altered neither the line crossings $\left(\mathrm{F}_{3,33}=0.44, \mathrm{P}=0.72\right)$ nor the rears $\left(\mathrm{F}_{3,33}=0.37, \mathrm{P}=\right.$ 0.77), suggesting that Zl-n-91 does not produce sedation at the dose of $0.5 \mathrm{mg} / \mathrm{kg}$, which is about 50 -fold higher than the dosage required for memory enhancement $(0.01 \mathrm{mg} / \mathrm{kg})$. In comparison, rolipram decreased locomotor activity at the same dose required for producing a memory-enhancing effect. ${ }^{44}$

\section{Discussion}

\section{The water-containing subpocket is PDE4 specific}

The structure superposition of the PDE families shows that the subpocket for binding of tetrahydrofuran of Zl-n-91 was named as the Q2 pocket in PDE4, ${ }^{39}$ the M-pocket in PDE9, ${ }^{45}$ and the PDE10 selectivity pocket. ${ }^{46}$ Since this pocket contains mainly the M-loop residues (Met352-Val365 of PDE4D2), we would use the term of the M-pocket in the later discussion. The M-pocket possesses variant amino acids and pocket sizes/shapes across PDE families, and may thus serve as a specific pocket for improvement of inhibitor selectivity of PDE families. The M-pocket of PDE4D is composed of Met337, Met357 and Val365 plus a water molecule, and can accommodate a five- or six-membered ring (Fig. 4B). The occupation of a water molecule in this pocket is apparently unique to PDE4, thus defining the PDE4 specific pocket. 
The PDE1B pocket is hydrophobic and consists of Met389, Phe392, Leu409, Val417, and Ser420 (Fig. 6A, drawn from PDB code 5B25). It has similar size to that of PDE4D and can adopt a hydrophobic group for improvement of selectivity, as shown by fit of the phenyl group of inhibitor ITI-214 to the pocket. ${ }^{47}$ There is no water found in the pocket.

The PDE2A pocket is composed of residues Tyr827, Phe830, and Met847 (Fig. 6B, drawn from 4HTX), but no water. ${ }^{48}$ It is rather small and does not appear to be capable of accommodating any substitution group. As the result, the catechol group of inhibitor BAY60-7550 has to orient to open surface in the PDE2A structure.

The M-pocket in the PDE3B structure is very small, contains no water, and is made up of Va1956, Leu984, and Leu987. Since Leu987 blocks the entry, the pocket is unlikely useful for inhibitor improvement. However, the Q1 subpocket corresponding to the CF2 binding site of PDE4D is large and fits well the cyclic hexane ring of inhibitor Merck1 (Fig. 6C, drawn from $1 \mathrm{SO} 2) .{ }^{49}$

The PDE5 pocket is constituted by the M-loop and a hydrophobic side of helix H14 (Ala779, Leu781, Val782, and Ala783, PDB code 3B2R). However, the M-loop is disordered in some of the PDE5 structures. ${ }^{50}$ No water was found in the PDE5-sildenafil structure, in which the M-loop has solid conformation and thus the whole pocket was defined. The ethoxyl tail of sildenafil fits the pocket (Fig. 6D). The replacement ethoxyl tail of sildenafil with propanoxyl group in vardenafil showed significant improvement on the binding affinity of vardenafil. ${ }^{51}$

The PDE9 pocket contains hydrophobic residues of Leu420, Leu421, Phe425, Phe441, and Val447, but no water (Fig. 6E, PDB code of 4Y8C). This pocket was suggested to serve as a specific pocket for selectivity enhancement of PDE9 inhibitors. ${ }^{44}$ It is slightly deeper and wider than that of PDE4D and may thus be useful for design of selective PDE9 inhibitors.

The PDE10 pocket is much deeper than those of the other PDE families and is pure hydrophobic pocket without water, as shown by binding of inhibitor triaryl imidazole to the PDE10 catalytic domain (PDB code 3HQW). ${ }^{46}$ It is apparently unique in terms of the accommodation of two five-membered rings, no stacking against the conserved Phe719, and no hydrogen bonds with invariant Gln716 (Fig. 6F).

In short, the M-pockets in the most PDE families are valuable for improvement of inhibitor selectivity and PDE4 is unique for existence of a bound water.

\section{Conclusion}

The water-containing subpocket is apparently unique to the PDE4 family and valuable for improvement of selectivity of PDE4 inhibitors. Our inhibitor (S)-Z1-n-91 forms a hydrogen bond with the water and shows great selectivity over other PDEs. 


\section{Acknowledgments}

We thank beamline X29A of Brookhaven National Laboratory for collection of diffraction data. This work was partially supported by US NIH GM59791 (H. K.), NIH HHSN275201700001C (H. T. Z.), and the Intramural Research Program of NIEHS (H. W.).

\section{Abbreviations: \\ PDE4 Phosphodiesterase 4 \\ COPD chronic obstructive pulmonary disease}

\section{References}

(1). Bender AT , and Beavo J (2006) Cyclic nucleotide phosphodiesterases: molecular regulation to clinical use. Pharmacol. Rev 58, 488-520.16968949

(2). Conti M , and Beavo J (2007) Biochemistry and physiology of cyclic nucleotide phosphodiesterases: Essential components in cyclic nucleotide signaling. Ann. Rev. Biochem 76, 481-511.17376027

(3). Maurice DH , Ke H, Ahmad F, Wang Y, Chung J , and Manganiello VC (2014) Advances in targeting cyclic nucleotide phosphodiesterases. Nat. Rev. Drug Discov 13, 290-314.24687066

(4). Kokkonen K , and Kass DA (2017) Nanodomain Regulation of Cardiac Cyclic Nucleotide Signaling by Phosphodiesterases. Annu. Rev. Pharmacol, Toxicol 57, 455-479.27732797

(5). Lipworth BJ (2005) Phosphodiesterase-4 inhibitors for asthma and chronic obstructive pulmonary disease. Lancet 365, 167-175.15639300

(6). Richter W , Menniti FS , Zhang HT and Conti M (2013) PDE4 as a target for cognition enhancement. Expert Opin. Ther. Targets 17, 1011-1027.23883342

(7). Salari-Sharif P , and Abdollahi M (2010) Phosphodiesterase 4 inhibitors in inflammatory bowel disease: a comprehensive review. Curr Pharm Des. 16, 3661-3667.21128899

(8). Kumar N , Goldminz AM ; Kim N , and Gottlieb AB (2013) Phosphodiesterase 4-targeted treatments for autoimmune diseases. BMC Med. 11, 96.23557064

(9). Beghè B , Rabe KF , and Fabbri LM (2013) Phosphodiesterase-4 inhibitor therapy for lung diseases. Am J Respir Crit Care Med. 188, 271-278.23656508

(10). Wittmann M , and Helliwell PS (2013) Phosphodiesterase 4 inhibition in the treatment of psoriasis, psoriatic arthritis and other chronic inflammatory diseases. Dermatol Ther (Heidelb). 3, $1-15.23888251$

(11). Ahluwalia J , Udkoff J , Waldman A , Borok J , and Eichenfield LF (2017) Phosphodiesterase 4 inhibitor therapies for atopic dermatitis: progress and outlook. Drugs. 77, 1389-1397.28681318

(12). Fusco FR , and Giampà C (2015) Phosphodiesterases as therapeutic targets for Huntington's disease. Curr Pharm Des. 21, 365-377.25159076

(13). Ramirez AD , and Smith SM (2014) Regulation of dopamine signaling in the striatum by phosphodiesterase inhibitors: novel therapeutics to treat neurological and psychiatric disorders. Cent Nerv Syst Agents Med Chem. 14, 72-82.25540976

(14). Hu W , Lu T , Chen A, Huang Y, Hansen R, Chandler LJ , and Zhang HT (2011) Inhibition of phosphodiesterase-4 decreases ethanol intake in mice. Psychopharmacology 218, 331339.21509503

(15). Garcia-Osta A , Cuadrado-Tejedor M , Garcia-Barroso C , Oyarzâbal J , and Franco R (2012) Phosphodiesterases as therapeutic targets for Alzheimer's disease. ACS Chem Neurosci. 3, 832844.23173065

(16). Heckman PR , Wouters C , and Prickaerts J (2015) Phosphodiesterase inhibitors as a target for cognition enhancement in aging and Alzheimer's disease: a translational overview. Curr Pharm Des. 21, 317-331.25159073 
(17). Prickaerts J , Heckman PRA , and Blokland A (2017) Investigational phosphodiesterase inhibitors in phase I and phase II clinical trials for Alzheimer's disease. Expert Opin Investig Drugs.26, 1033-1048.

(18). Pinner NA , Hamilton LA and Hughes A (2012) Roflumilast: a phosphodiesterase-4 inhibitor for the treatment of severe chronic obstructive pulmonary disease. Clin. Ther 34, 56-66.22284994

(19). Poole RM and Ballantyne AD (2014) Apremilast: first global approval. Drugs. 74, 825837.24797159

(20). Nazarian R and Weinberg JM (2009). AN-2728, a PDE4 inhibitor for the potential topical treatment of psoriasis and atopic dermatitis. Curr. Opin. Investig. Drugs 10 1236-1242.

(21). Contreras S , Milara J , Morcillo E , and Cortijo J (2017) Selective Inhibition of Phosphodiesterases 4A, B, C and D isoforms in chronic respiratory diseases: current and future evidences. Curr Pharm Des. 23, 2073-2083.28201975

(22). Pearse DD , and Hughes ZA (2016) PDE4B as a microglia target to reduce neuroinflammation. Glia. 64, 1698-709.27038323

(23). Clapcote SJ (2017) Phosphodiesterase-4B as a therapeutic target for cognitive impairment and obesity-related metabolic diseases. Adv Neurobiol. 17, 103-131.28956331

(24). Chauret N , Guay D , Li C , Day S , Silva J , Blouin M , Ducharme Y, Yergey JA , and NicollGriffith DA (2002) Improving metabolic stability of phosphodiesterase-4 inhibitors containing a substituted catechol: prevention of reactive intermediate formation and covalent binding, Biooorg. Med. Chem. Lett 12, 2149-2152.

(25). Harris CS , Hennequin LF , Kettle JG , and Willerval OA (2005) Selective alkylation of a 6,7dihydroxyquinazoline. Tetrahedron Lett. 45, 7715-7719.

(26). Schmidt AH , Kircher G, Kuenz C , Wahl S , and Hendriok MW (1995) Regioselective Annulation of 5-(1-Alkenyl)- and 5-Vinyl-1,3-benzodioxoles with 3-Chloro-3-cyclobutene-1,2dione. Synthesis of 3,4-Dihydrocyclobuta[5,6]- naphtho[2,3-d][1,3] dioxole-1,2-diones and Cyclobuta[6,7]naphtho[2,3-d] [1,3]dioxole-1,2-diones. J. Org. Chem. 12, 3890-3894.

(27). Spera D , Cabrera G , Fiaschi R , Carlson KE , Katzenellenbogen JA, and Napolitano E (2004) Estradiol derivatives bearing sulfur-containing substituents at the $11 \beta$ or $7 a$ positions: versatile reagents for the preparation of estrogen conjugates. Bioorg. Med. Chem 16, 4393-4401.

(28). Huai Q, Wang H, Sun Y, Kim HY, Liu Y, and Ke H (2003) Three dimensional structures of PDE4D in complex with roliprams and implication on inhibitor selectivity. Structure 11, 865873.12842049

(29). Huai Q, Wang H, Zhang W , Colman RW , Robinson H , and Ke H (2004) Crystal structure of phosphodiesterase 9 in complex with inhibitor IBMX. Proc. Natl. Acad. Sci. USA 101, 96249629.15210993

(30). Wang H , Liu Y, Chen Y, Robinson H , and Ke H (2005) Multiple elements jointly determine inhibitor selectivity of cyclic nucleotide phosphodiesterases 4 and 7. J. Biol. Chem 280, 3094930955.15994308

(31). Wang H , Liu Y , Hou J , Zheng M , Robinson H , and Ke H (2007) Structural insight into substrate specificity of phosphodiesterase 10. Proc. Natl. Acad. Sci. USA, 104, 5782 5787.17389385

(32). Wang H , Yan Z, Yang S , Cai J , Robinson H , and Ke H (2008) Kinetic and structural studies of phosphodiesterase-8A and implication on the inhibitor selectivity. Biochemistry 47, 12760 12768.18983167

(33). Otwinowski Z , and Minor W (1997) Processing of X-ray diffraction data collected in oscillation mode. Methods Enzymol. 276, 307-326.

(34). Navaza J , and Saludjian P (1997) AMoRe: an automated molecular replacement program package. Methods Enzymol. 276, 581-594.27799116

(35). Emsley P , Lohkamp B , Scott WG, and Cowtan K (2010) Features and development of Coot. Acta Cryst. 66, 486-501.

(36). Winn MD , Murshudov GN and Papiz MZ (2003) Macromolecular TLS refinement in REFMAC at moderate resolutions. Method Enz. 374, 300-321 
(37). Li LX , Cheng YF , Lin HB , Wang C , Xu JP , and Zhang HT (2011) Prevention of cerebral ischemia-induced memory deficits by inhibition of phosphodiesterase- 4 in rats. Metab. Brain Dis 26, 37-47.21327879

(38). Zhang HT , Huang Y, Masood A , Stolinski LR , Li Y, Zhang L , Dlaboga D , Jin SL Conti M , and O'Donnell JM (2008) Anxiogenic-Like Behavioral Phenotype of Mice Deficient in Phosphodiesterase 4B (PDE4B). Neuropsychopharmacology 33, 1611-1623.17700644

(39). Card GL , England BP, Suzuki Y, Fong D, Powell B , Lee B , Luu C, Tabrizizad M , Gillette S , Ibrahim PN , Artis DR, Bollag G, Milburn MV Kim SH, Schlessinger J , and Zhang KY (2004) Structural basis for the activity of drugs that inhibit phosphodiesterases. Structure 12, 2233-2247.15576036

(40). Ke H , and Wang H (2007) Crystal structures of phosphodiesterases and implications on substrate specificity and inhibitor selectivity. Curr. Top. Med. Chem 7, 391-403.17305581

(41). Zheng S , Kaur G, Wang H , Li M , Macnaughtan M , Yang X Reid S , Prestegard J , Wang B , and Ke H (2008) Design, synthesis, and structure-activity relationship, molecular modeling, and NMR studies of a series of phenyl alkyl ketones as highly potent and selective phosphodiesterase-4 inhibitors. J. Med. Chem 51, 7673-7688.19049349

(42). Ouagued M , Martin-Chouly CA , Brinchault G, Leportier-Comoy C, Depincé A , Bertrand C, Lagente V , Belleguic C , and Pruniaux MP (2005) The novel phosphodiesterase 4 inhibitor, CI-1044, inhibits LPS-induced TNF-alpha production in whole blood from COPD patients. Pulm. Pharmacol. Ther 18, 49-54.15607127

(43). Wang H , Peng M , Chen Y, Geng J , Robinson H , Houslay M , Cai D , J. , and Ke H (2007) Structures of the four subfamilies of phosphodiesterase-4 provide insight into the selectivity of their inhibitors. Biochem. J 408, 193-201.17727341

(44). Zhang HT , Crissman AM, Dorairaj NR, Chandler LJ , and O'Donnell JM (2000) Inhibition of Cyclic AMP Phosphodiesterase (PDE4) Reverses Memory Deficits Associated with NMDA Receptor Antagonism. Neuropsychopharmacology, 23, 198-204.10882846

(45). Huang M , Shao Y , Hou J , Cai W , Liang B , Huang Y, Li Z, Wu Y , Zhu X, Liu P, Wan Y, Ke H , and Luo HB (2015) Structural Asymmetry of Phosphodiesterase-9A and a Unique Pocket for Selective Binding of a Potent Enantiomeric Inhibitor. Mol. Pharmacol. 88, 836845.26316540

(46). Verhoest PR, Chapin DS , Corman M , Fonseca K , Harms JF , Hou X, Marr ES Menniti FS , Nelson F, O'Connor R, Pandit J , Proulx-Lafrance C , Schmidt AW , Schmidt CJ , Suiciak JA, and Liras S . (2009) Discovery of a novel class of phosphodiesterase 10A inhibitors and identification of clinical candidate 2-[4-(1-methyl-4-pyridin-4-yl-1H-pyrazol-3-yl)phenoxymethyl]-quinoline (PF-2545920) for the treatment of schizophrenia. J. Med. Chem. 52, 5188-5196.19630403

(47). Li P , Zheng H , Zhao J , Zhang L, Yao W , Zhu H , Beard JD , Ida K , Lane W , Snell G, Sogabe S , Heyser CJ , Snyder GL, Hendrick JP, Vanover KE , Davis RE, and Wennogle LP (2016) Discovery of Potent and Selective Inhibitors of Phosphodiesterase 1 for the Treatment of Cognitive Impairment Associated with Neurodegenerative and Neuropsychiatric Diseases. J. Med. Chem. 59, 1149-1164.26789933

(48). Zhu J , Yang Q , Dai D , and Huang Q (2013) X-ray crystal structure of phosphodiesterase 2 in complex with a highly selective, nanomolar inhibitor reveals a binding-induced pocket important for selectivity. J. Am. Chem. Soc. 135, 11708-11711.23899287

(49). Scapin G, Patel SB , Chung C, Varnerin JP , Edmondson SD, Mastracchio A, Parmee ER , Singh SB , Becker JW, Van der Ploeg LH, and Tota MR (2004) Crystal structure of human phosphodiesterase 3B: atomic basis for substrate and inhibitor specificity. Biochemistry 43, 6091-6100.15147193

(50). Wang H, Liu Y, Huai Q, Cai J , Zoraghi R , Francis SH , Corbin JD , Robinson H , Xin Z , Lin $\mathrm{G}$, and Ke H (2006) Multiple conformations of phosphodiesterase-5: implications for enzyme function and drug development. J. Biol. Chem. 281, 21469-21479.16735511

(51). Wang H , Ye M , Robinson H , Francis SH , and Ke H , (2008) Conformational variations of both PDE5 and inhibitors provide the structural basis for the physiological effects of vardenafil and sildenafil. Mol. Pharmacol. 73, 104-110.17959709 


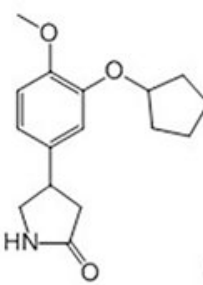

Rolipram

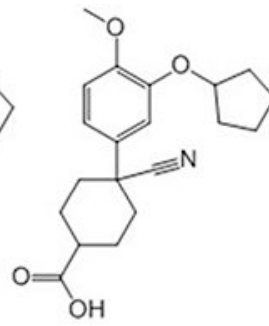

Cilomilast

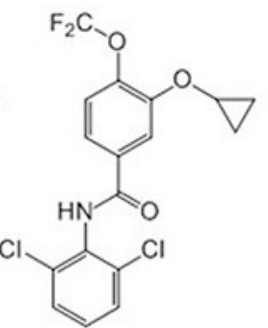

Roflumilast<smiles>CC(C)CC(=O)c1ccc(OC(F)(F)F)c(OC2CCOC2)c1</smiles>

Zl-n-91

Fig. 1.

Rolipram-based PDE4 inhibitors 

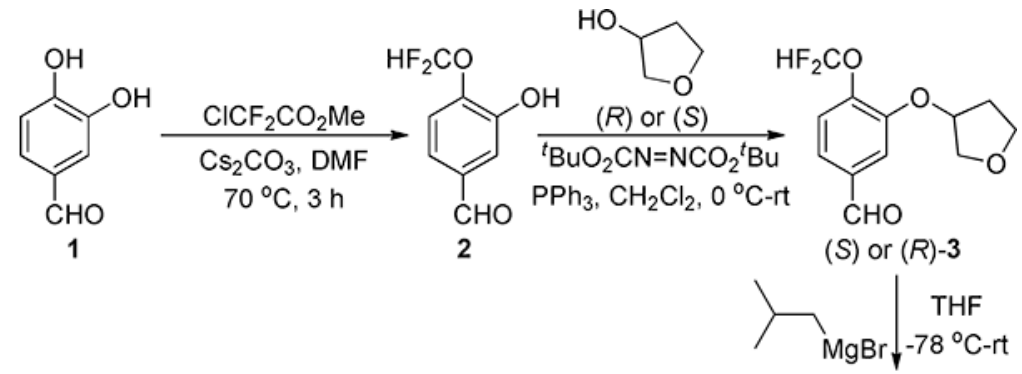

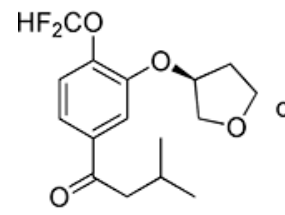

(S) -ZI-n-91

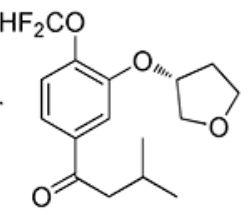

(R)-ZI-n-91

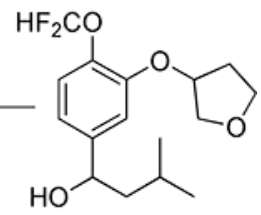

$(S)$ or $(R)-4$

Fig. 2.

Synthesis of $(S)$ or $(R)-Z 1-n-91$ 


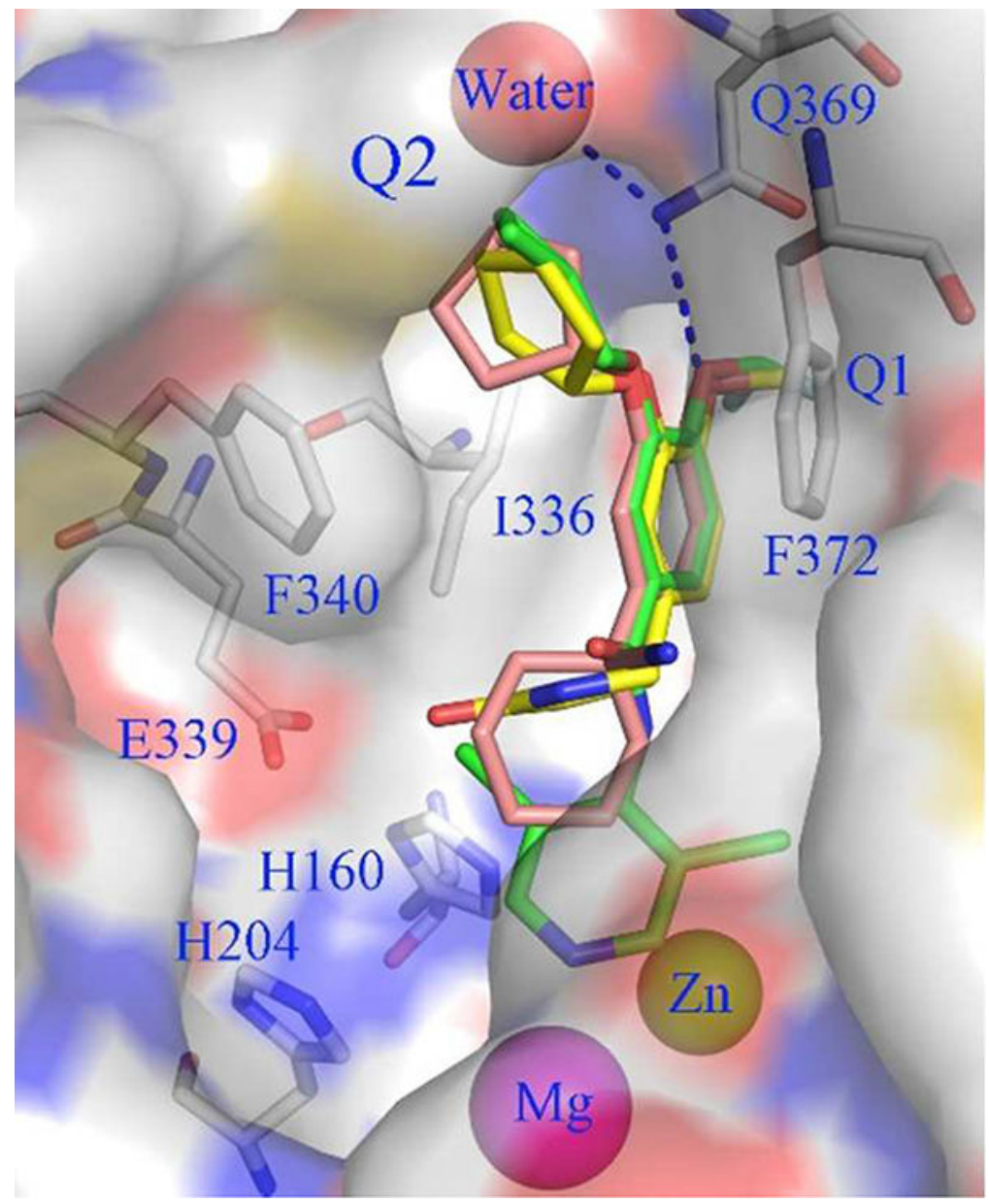

Fig. 3.

Surface presentation on the active site of PDE4D. The binding pocket can be divided into a metal site, an open space (left around H204 and E339), and two inhibitor subpockets (Q1 and Q2), as shown by PDE4 inhibitors of rolipram (yellow sticks), cilomilast (salmon), and roflumilast (green). Dotted lines represent hydrogen bonds. 

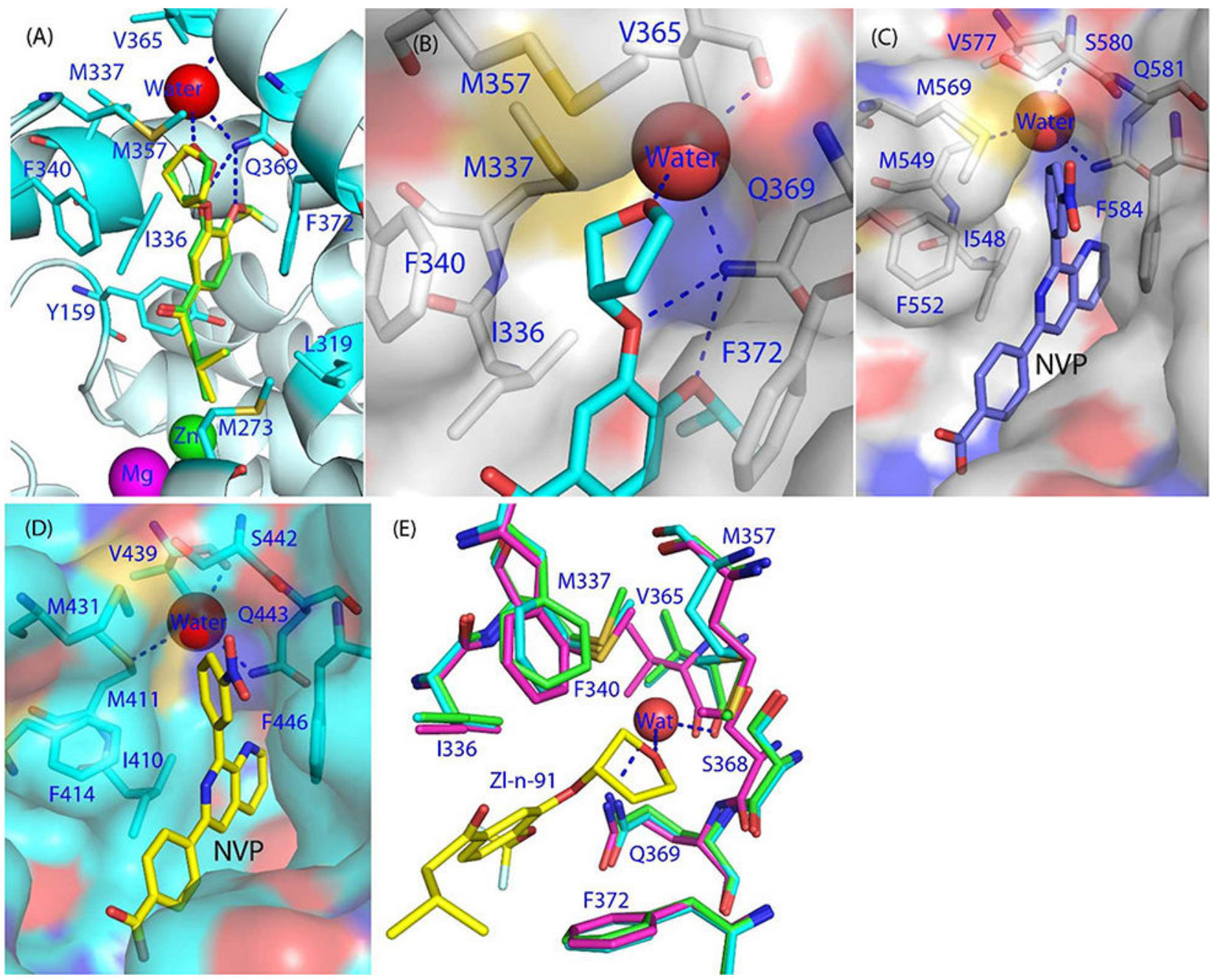

Fig. 4.

The water-containing subpockets of PDE4s. Dotted lines represent hydrogen bonds. (A) Ribbon model on binding of $(S)$ - and $(R)$ - Zl-n-91 (green and yellow sticks) to the PDE4D2 pocket. (B) A close view on the interaction of (S)-Zl-n-91 (cyan sticks) with the bound water in PDE4D2. (C) A bound water in the PDE4A pocket. NVP (blue sticks) is a selective PDE4 inhibitor. (D) A bound water in the PDE4B pocket. (E) Superposition of the water-binding pocket of PDE4s. Residues are shown in colors of green (4D), cyan (4B), and pink (4A). Only PDE4D residues are labeled. The residues of PDE4A and PDE4B can be referred to Figs. 4C \& 4D. 

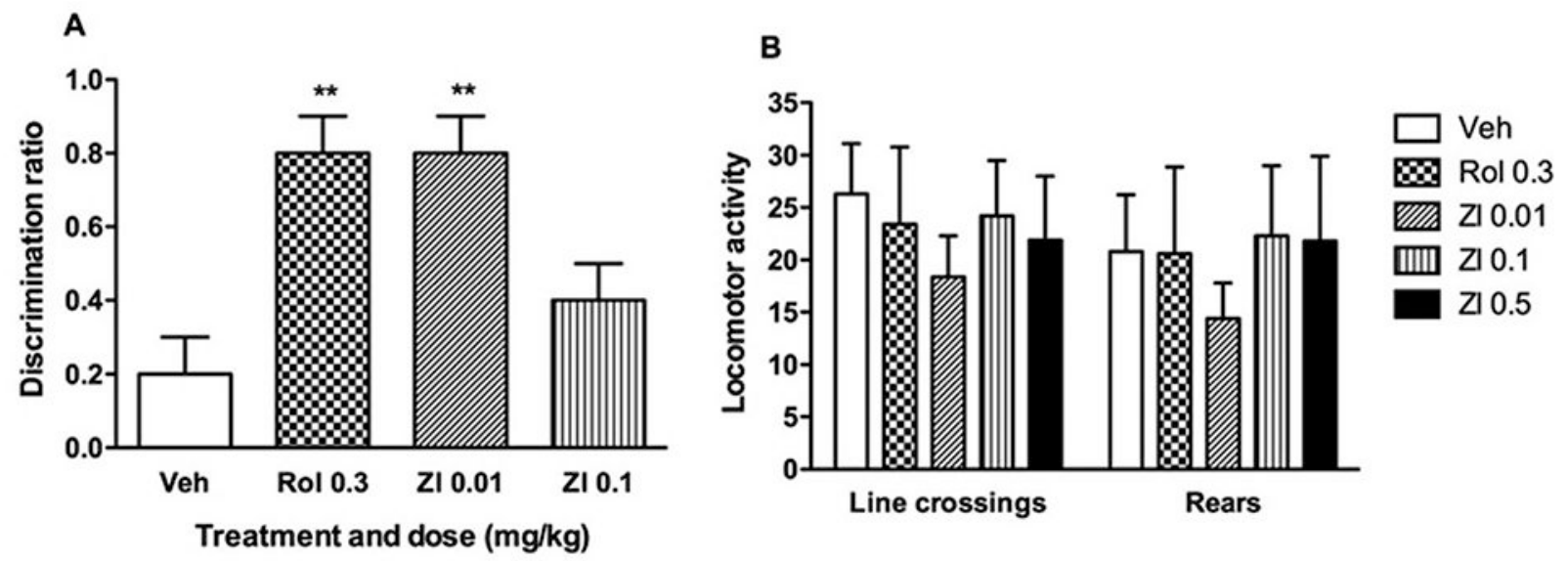

Fig. 5.

Effects of Z1-n-91 on memory. (A) Zl-n-91 (Zl) and rolipram (Rol) enhanced memory in the object recognition test of mice. The discrimination ratio (Tn-Tf)/(Tn+Tf) was defined as the difference of time spent in exploring the novel (Tn) and familiar (Tf) objects. An increase in discrimination ratio is indicative of memory enhancement. $\mathrm{Zl}$, Rol, or vehicle (Veh) was injected (i.p.) 30 min prior to the test. $\mathrm{n}=8-9$; ** $\mathrm{P}<0.01$ vs. Veh. (B) Effect of Zl-n-9 and rolipram on locomotor activity in the open-field test of mice. After thirty minutes of injection of $\mathrm{Zl}$, Rol, or Veh, line crossings and rears were recorded for 5 min. Bars are means \pm S.E. $n=8-9$. All data were analyzed using one-way ANOVA, followed by post hoc Dunnett's tests. Statistical significance was accepted at $\mathrm{p}<0.05$. 

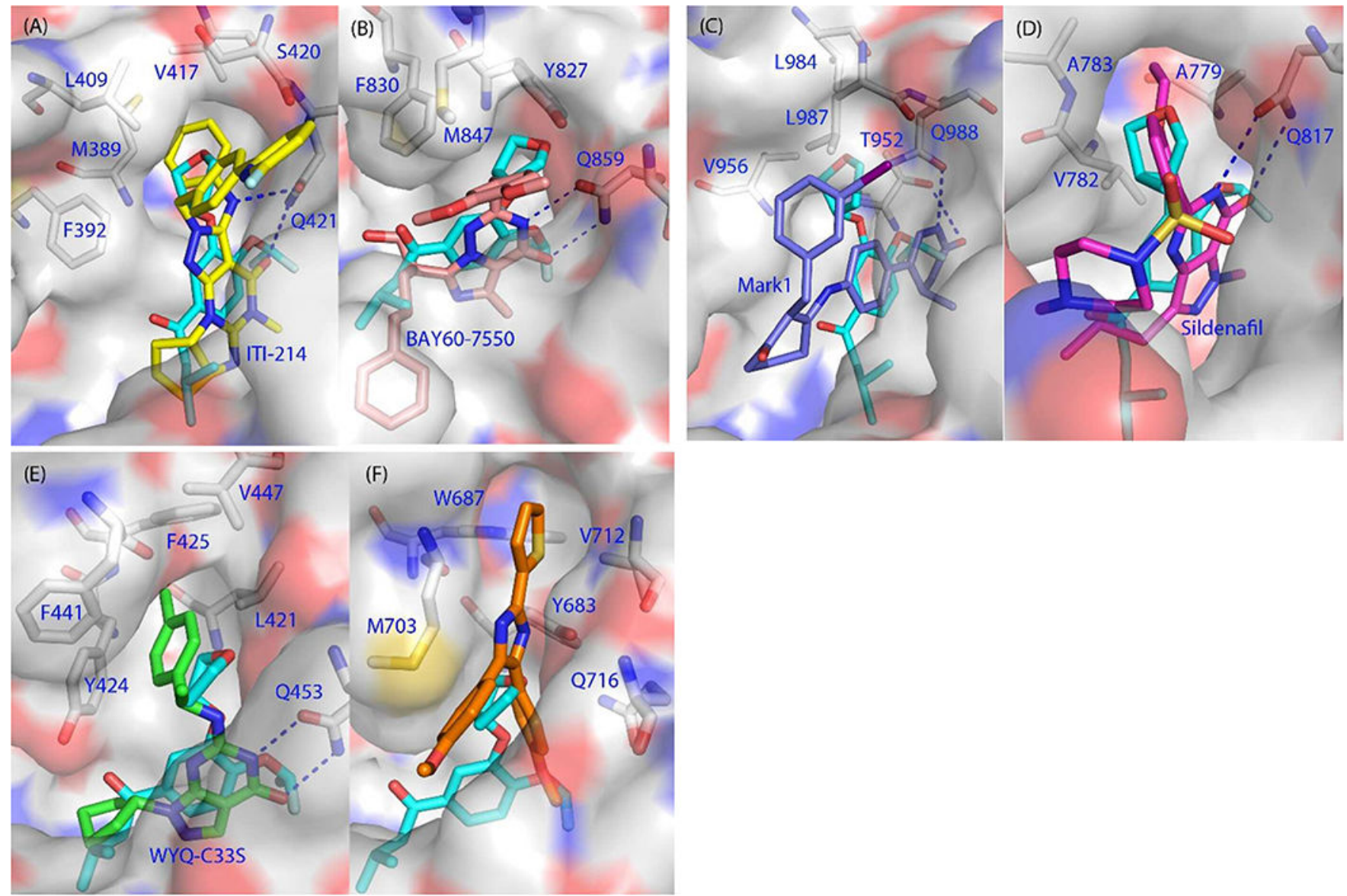

Fig. 6.

Inhibitor binding to the M-pockets of the PDE families. Dotted lines represent hydrogen bonds. PDE4 inhibitor (S)-Zl-n-91 is shown as cyan sticks in all the drawings. (A) Surface presentation on binding of inhibitor ITI-214 (yellow sticks, PDB code 5B25) to PDE1B. (B) Binding of inhibitor BAY 60-7550 (salmon, PDB code 4HTX) to PDE2A. (C) Binding of inhibitor Merck1 (light blue, PDB code 1SO2) to PDE3B. (D) Binding of sildenafil (pink, PDB code 3B2R) to PDE5. (E) Binding of WYQ-C33S (green,PDB code 4Y8C) to PDE9. (F) Binding of inhibitor triaryl imidazoes (brown, PDB code 3HQW)to PDE10. 
Table 1.

Statistics on diffraction data and structure refinement

\begin{tabular}{|c|c|c|}
\hline Data collection & PDE4D-( $R)-Z 1-\mathrm{n}-91$ & PDE4D2-( $S$ ) Z1-n-91 \\
\hline Space group & $\mathrm{P} 2_{1} 2_{1} 2_{1}$ & $\mathrm{P} 2_{1} 2_{1} 2_{1}$ \\
\hline Unit cell (a, b. c, ̊̊) & $59.5,79.9,163.4$ & $5.8 .9,80.2,163.7$ \\
\hline Resolution $(\AA)$ & 1.8 & 1.6 \\
\hline Unique reflections & 69,331 & 95,949 \\
\hline Total measurements & 918,592 & $1,207,849$ \\
\hline Completeness (\%) & $94.6(67.2)^{*}$ & $93.1(59.2)$ \\
\hline Average E $\sigma$ & $8.8(2.6) *$ & $10.5(2.1)$ \\
\hline Rmerge & $0.068(0.34)^{*}$ & $0.068(0.40)$ \\
\hline \multicolumn{3}{|l|}{ Structure Refinement } \\
\hline R-factor & 0.193 & 0.206 \\
\hline R-free & $0.214(5.0 \%)^{*}$ & $0.224(5.0 \%)^{*}$ \\
\hline Resolution $(\AA)$ & $50-1.8$ & $50-1.6$ \\
\hline \multicolumn{3}{|l|}{ RMS deviation for } \\
\hline Bond $(\AA)$ & 0.005 & 0.005 \\
\hline Angle & $0.95^{\circ}$ & $0.93^{\circ}$ \\
\hline \multicolumn{3}{|l|}{ Average B-factor $\left(\AA^{2}\right)$} \\
\hline Protein & $26.8(5271)^{\xi}$ & $28.2(5271) \mathcal{g}$ \\
\hline Inhibitor & $24.8(44)$ & $25.5(44)$ \\
\hline $\mathrm{Zn}$ & $19.7(2)$ & $19.0(2)$ \\
\hline $\mathrm{Mg}$ & $18.9(3)$ & $18.0(3)$ \\
\hline Water & $34.1(388)$ & $37.5(384)$ \\
\hline \multicolumn{3}{|c|}{ Remachandran Plot (\%) } \\
\hline Most favored & 92.5 & 93.0 \\
\hline Allowed & 7.5 & 6.8 \\
\hline Generally allowed & 0 & 0.2 \\
\hline \multicolumn{3}{|c|}{ The numbers in parentheses are for the highest resolution shell. } \\
\hline
\end{tabular}


Table 2.

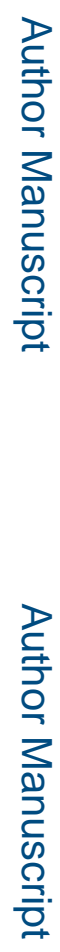

Inhibition of PDEs by (S)-Z1-n-91

\begin{tabular}{|l|l|}
\hline PDEs & $\mathrm{IC}_{50}(\mu \mathrm{M})$ \\
\hline PDE4D2 (1-507) & $0.012 \pm 0.002$ \\
\hline PDE4B2B (152-528) & $0.020 \pm 0.004$ \\
\hline PDE2A3 (580-941) & $83 \pm 12$ \\
\hline PDE3A1 (665-1141) & $46 \pm 3$ \\
\hline PDE5A1 (535-860) & $130 \pm 23$ \\
\hline PDE7A1 (130-482) & $17 \pm 3$ \\
\hline PDE8A1 (480-820) & $8.6 \pm 0.7$ \\
\hline PDE9A1 (181-506) & $84 \pm 10$ \\
\hline PDE10A2 (448-789) & $16 \pm 1$ \\
\hline PDE11A4 (563-934) & $23 \pm 4$ \\
\hline
\end{tabular}

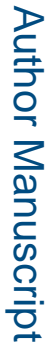

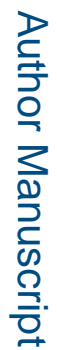

Biochemistry. Author manuscript; available in PMC 2018 August 13. 
Table 3.

Pharmacokinetic properties of Z1-n-91

\begin{tabular}{|l|l|l|l|l|l|l|l|l|l|}
\hline $\begin{array}{l}\text { Dosing } \\
\text { route }\end{array}$ & $\begin{array}{l}\mathrm{T}_{1 / 2} \\
\text { Range(h) }\end{array}$ & $\begin{array}{l}\mathrm{T}_{1 / 2} \\
(\mathrm{~h})\end{array}$ & $\begin{array}{l}\mathrm{T}_{\max } \\
(\mathrm{h})\end{array}$ & $\begin{array}{l}\mathrm{C}_{\max } \\
(\mathrm{ng} / \mathrm{mL})\end{array}$ & $\begin{array}{l}\mathrm{AUC}_{0-\mathrm{t}} \\
(\mathrm{ng} / \mathrm{h} / \mathrm{mL})\end{array}$ & $\begin{array}{l}\mathrm{AUC}_{0-\mathrm{m}} \\
(\mathrm{ng} / \mathrm{h} / \mathrm{mL})\end{array}$ & $\begin{array}{l}\mathrm{V}_{\mathrm{z}}(\mathrm{F}) \\
(\mathrm{L} / \mathrm{kg})\end{array}$ & $\begin{array}{l}\mathrm{CL}(\mathrm{F}) \\
(\mathrm{L} / \mathrm{h} / \mathrm{kg})\end{array}$ & $\mathrm{F}(\%)$ \\
\hline PO & $4-8$ & 1.35 & 2 & 96.5 & 241 & 244 & 39.9 & 20.5 & 33.3 \\
\hline $\begin{array}{l}\text { Dosing } \\
\text { route }\end{array}$ & $\begin{array}{l}\mathrm{T}_{1 / 2} \\
\text { Range(h) }\end{array}$ & $\begin{array}{l}\mathrm{T}_{1 / 2} \\
(\mathrm{~h})\end{array}$ & $\mathrm{C}_{0}$ & $\mathrm{CL}$ & $\begin{array}{l}\mathrm{AUC} \\
(\mathrm{ng} / \mathrm{h} / \mathrm{mL})\end{array}$ & $\begin{array}{l}\mathrm{AUC} \mathrm{A}_{0-\mathrm{m}} \\
(\mathrm{ng} / \mathrm{h} / \mathrm{mL})\end{array}$ & $\mathrm{Vss}$ & $\begin{array}{l}\text { MRTinf } \\
(\mathrm{h})\end{array}$ & \\
\hline IV & $0.5-4$ & 0.62 & 6030 & 6.87 & 725 & 728 & 2.31 & 0.34 & \\
\hline
\end{tabular}

